\title{
Decreased brain and muscle ARNT-like protein 1 expression mediated the contribution of hyperandrogenism to insulin resistance in polycystic ovary syndrome
}

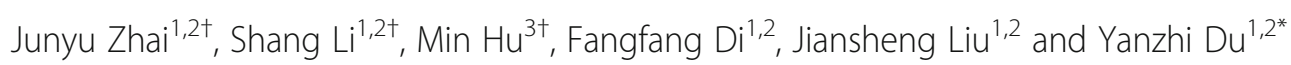

\begin{abstract}
Background: The interface between environmental risk factors and genetic factors could contribute to the pathogenesis of hyperandrogenism and insulin resistance in polycystic ovary syndrome (PCOS); however, the underlying complex mechanism remains to be elucidated.
\end{abstract}

Methods: We used dehydroepiandrosterone (DHEA)-induced PCOS-like rat model to measure circadian clock genes and insulin resistance-related genes. Additionally, we performed in vitro experiments in mature adipocytes to verify the molecular mechanisms.

Results: DHEA-induced PCOS-like rats exhibited insulin resistance and arrhythmic expression of circadian clock genes in the liver and adipose tissues, particularly showing decreased brain and muscle ARNT-like protein 1 (BMAL1) expression. In addition, hyperandrogenism gave rise to negative regulation of BMAL1 expression to nicotinamide phosphoribosyltransferase and sirtuin 1, which further inhibited downstream glucose transporter type 4, leading to insulin resistance in mature adipocytes, which was consistent with our previous results in HepG2 cells.

Conclusions: Decreased BMAL1 expression in the liver and adipose played a potentially novel role in the contribution of hyperandrogenism to insulin resistance, which might be a possible mechanism accounting for the pathogenesis of PCOS.

Keywords: BMAL1, Hyperandrogenism, Insulin resistance, PCOS, Circadian clock

\section{Background}

Polycystic ovary syndrome (PCOS) is a heterogeneous disorder characterized by hyperandrogenism, irregular menstrual cycle, and polycystic ovaries [1]. It is the most

\footnotetext{
*Correspondence: duyz@sjtu.edu.cn

†Junyu Zhai, Shang Li and Min Hu contributed equally to this work.

${ }^{1}$ Center for Reproductive Medicine, Ren Ji Hospital, School of Medicine, Shanghai Jiao Tong University, 845 Lingshan Road, Shanghai 200135, China

${ }^{2}$ Shanghai Key Laboratory for Assisted Reproduction and Reproductive Genetics, Shanghai 200135, China

Full list of author information is available at the end of the article
}

common endocrine disease affecting $6-20 \%$ of women of reproductive age, with a varied pathogenesis ranging from genetic to environmental factors. The association between hyperandrogenism and insulin resistance leads to the pathogenesis of PCOS; however, the specific mechanism underlying the contribution of hyperandrogenism to insulin resistance is still not fully understood.

Insulin resistance, the state in which more insulin is required to maintain glucose homeostasis, is a common phenotype of PCOS patients and contributes to the

C C The Author(s). 2020 Open Access This article is licensed under a Creative Commons Attribution 4.0 International License, which permits use, sharing, adaptation, distribution and reproduction in any medium or format, as long as you give appropriate credit to the original author(s) and the source, provide a link to the Creative Commons licence, and indicate if changes were made. The images or other third party material in this article are included in the article's Creative Commons licence, unless indicated otherwise in a credit line to the material. If material is not included in the article's Creative Commons licence and your intended use is not permitted by statutory regulation or exceeds the permitted use, you will need to obtain permission directly from the copyright holder. To view a copy of this licence, visit http://creativecommons.org/licenses/by/4.0/. The Creative Commons Public Domain Dedication waiver (http://creativecommons.org/publicdomain/zero/1.0/) applies to the data made available in this article, unless otherwise stated in a credit line to the data. 
pathogenesis of PCOS. Approximately $85 \%$ of PCOS patients, particularly those who are overweight, have insulin resistance [2]. Insulin signaling is complicated, and any changes in the process, such as phosphorylation of insulin receptors, insulin receptor substrate-1, or the phosphoinositide-3-kinase (PI3K)/protein kinase B (AKT) pathway and the subsequent expression of glucose transporter 4 (GLUT4), may play a role in the insulin-resistant state of PCOS [3]. Studies have indicated that multiple environmental risk factors such as continuous darkness or illumination, excessive food intake, lack of exercise, and environmental endocrine disruptors could contribute to the onset of PCOS [4].

Circadian clock is the biochemical oscillator that synchronizes human activities with solar time, and this internally synchronized circadian clock enables humans to coordinate their biology and behavior with daily environmental changes corresponding to the day-night cycle. The circadian clock of each tissue synchronizes endogenous and exogenous signals to regulate the transcriptional activity throughout the day in a tissuespecific manner through the regulation of circadian clock genes [5]. Circadian locomotor output cycles kaput (CLOCK) and brain and muscle ARNT-like protein 1 (BMAL1) heterodimers bind to cis-acting E-box elements to drive the transcription of cryptochrome (CRY) and period $(P E R)$, which subsequently return to the nucleus and repress their own transcription [6].

Circadian misalignment, a typical feature of jet lag and shift work, induces insulin resistance in humans [7], and elevates serum glucose and insulin levels in rats [8]. Mutations of the core circadian clock genes have been linked to the characteristics of the metabolic syndrome commonly associated with PCOS [9]. Our previous work has also presented that the arrhythmic expressions of circadian clock genes due to constant darkness result in the metabolic and reproductive hallmarks of PCOS in rats [10]. Reportedly, sleep disturbances are doubled in PCOS patients, and obstructive sleep apnea is a common feature of these patients. An appropriately classical pharmacological treatment combined with melatonin is recommended for PCOS patients to restore their endocrine-metabolic and reproductive functions [11]. More importantly, hyperandrogenism, the primary PCOS inducer, disrupts circadian organization in female rats [12] and female mice [13]. Although morning circadian misalignment is suggested associated with worse insulin sensitivity and higher serum free testosterone levels in girls with PCOS and obesity [14], the association among hyperandrogenism, metabolic homeostasis, and circadian clock function at the cellular level remains poorly understood.

In this study, we aimed to investigate the role of circadian clock genes in the contribution of hyperandrogenism to insulin resistance, which might also aid in determining appropriate interventions for PCOS patients.

\section{Materials and methods PCOS-like rat model}

Female Sprague-Dawley (SD) rats (3 weeks old) were divided into five rats per cage under constant environmental conditions with a 12-h light-dark cycle. Food and water were provided ad libitum. The female rats in the dehydroepiandrosterone (DHEA) group $(N=30)$ received daily injections (s.c.) of DHEA (Langchem, Shanghai, China) (6 mg/100 g body weight) for 4 weeks, starting at the age of 3 weeks. The control group ( $N=$ 30) was injected the same volume of peanut oil. The PCOS-like rat model was selected according to previously reported criteria including estrous cycle, glucose tolerance test (GTT), and hematoxylin and eosin (HE) staining of ovarian tissues [3]. The body weight was detected every week from 0 to 4 weeks during the model building. After 4-week treatment, six rats from each group were killed every $5 \mathrm{~h}$ from ZT0 to ZT20. ZT0 was defined as 7:30 a.m. according to the lights-on time of the animal laboratory. Adipose, liver, ovaries, and serum were collected immediately after decapitation and stored for further analyses.

\section{GTT}

After the 4-week treatment, female rats in the control and DHEA groups were kept under fasting for $16 \mathrm{~h}(5$ p.m. to 9 a.m.) with free access to drinking water. Then, D-glucose $(2.0 \mathrm{~g} / \mathrm{kg}$ body weight) was intraperitoneally injected into each rat. Blood glucose levels were measured before and 30, 60, 90, and $120 \mathrm{~min}$ after the Dglucose injection using an Accu-Chek glucose monitor (Roche Diagnostics Corp., Basel, Switzerland).

\section{HE staining}

Rat ovaries were fixed with $4 \%$ paraformaldehyde and embedded in paraffin. Tissue sections (thickness, $5 \mu \mathrm{m}$ ) were prepared, followed by deparaffinization and rehydration through a graded ethanol series. Sections then were stained in hematoxylin for $5 \mathrm{~min}$ and differentiated by hydrochloric acid for $30 \mathrm{~s}$. Finally the sections were incubated in eosin for $2 \mathrm{~min}$ before covering the slide and visualizing under a microscope (Zeiss, Oberkochen, Germany).

\section{Cell culture}

3T3-L1 preadipocytes were cultured in Dulbecco's modified eagle medium (DMEM)/high glucose (Gibco, Grand Island, NY, USA) supplemented with $10 \%$ fetal bovine serum (FBS) (Gibco) and 1\% penicillin-streptomycinneomycin (Gibco) at $37^{\circ} \mathrm{C}$ in a humidified 
atmosphere with $5 \% \mathrm{CO}_{2}$. 3T3-L1 preadipocytes were induced to differentiate into mature adipocytes as previously described [15]. Oil red O staining (Sigma Chemical, St. Louis, MO, USA) was used to identify the mature adipocytes.

\section{Small interfering (si) RNA knockdown}

A mixture of siRNA (50 pmol) and RNAiMAX (Invitrogen, Carlsbad, CA, USA) $(9 \mu \mathrm{l})$ in OPTI-MEM $(250 \mu \mathrm{l})$ was added to each well, and target genes were detected after $48 \mathrm{~h}$. The specific sequences of the targeted genes were as follows:

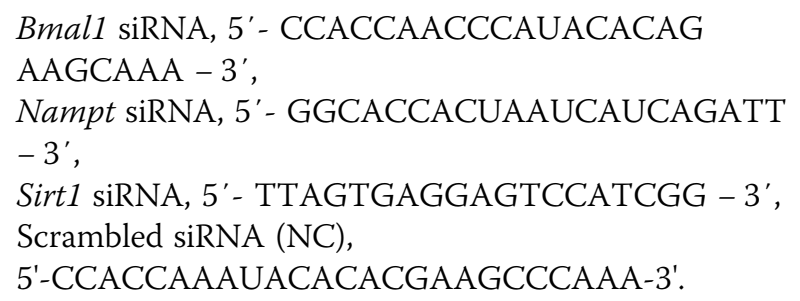

\section{Glucose uptake}

Glucose uptake in cell lines was measured after insulin stimulation (100 nM for $20 \mathrm{~min}$ ) using a Glucose Uptake-Glo $^{\text {tw }}$ Assay (Promega, Wisconsin, USA) according to manufacturer's instructions.

\section{Enzyme-linked immunosorbent assay (ELISA)}

The rats serum were detected using ELISA kits for mouse/rat leptin quanticine (R\&D Systems, MN, USA), rat total adiponectin/Acrp30 quantikine (R\&D), testosterone (Cayman Chemical, Michigan, USA), rat luteinizing hormone (LH) (MyBiosource, San Diego, USA), and rat follicle-stimulating hormone (FSH) (Biomatik, Ontario, Canada). Nicotinamide adenine dinucleotide $\left(\mathrm{NAD}^{+}\right)$levels in mature adipocytes were determined using an NAD/NADH Assay Kit (Abcam, Cambridge, UK). All procedures were performed according to manufacturer's instructions.

\section{Western blot}

Approximately $40 \mu \mathrm{g}$ of protein was separated on a $10 \%$ SDS gel, following which it was transferred to a nitrocellulose membrane. The nonspecific binding sites of the nitrocellulose membrane were blocked using 5\% nonfat milk and then incubated with anti-BMAL1 (Abcam) (1: 1000), anti-T-AKT (Cell Signaling Technology, Danvers, MA, USA) (1:1000), anti-P-AKT (Ser473) (Cell Signaling Technology) (1:1000), and anti-GAPDH (Abcam) (1: 5000) antibodies at $4{ }^{\circ} \mathrm{C}$ overnight. After washing, the blot was incubated and diluted with the corresponding peroxidase-conjugated secondary antibodies for $1 \mathrm{~h}$ at room temperature. Finally, the protein signals were detected using the enhanced chemiluminescent detection system (Millipore, Billerica, MA), and the ratio of a target protein to that of the intensity of GAPDH was obtained as each target protein level.

\section{Real-time quantitative polymerase chain reaction (RT- qPCR)}

Total RNA was extracted from cells and rat tissues using an animal total RNA isolation kit (FOREGENE, Chengdu, China) and then reverse transcribed into cDNA (TAKARA, Dalian, China). RT-qPCR was used to detect the abundance of target genes. Following this, the results were analyzed through the $\Delta \Delta \mathrm{Ct}$ method using $\beta$-Actin as the housekeeping gene. The primer sequences targeted genes are presented in Table 1.

\section{Statistical analysis}

Statistical analyses were performed using the SPSS software package (version 22, SPSS Inc., Chicago, USA). For normally distributed data, we used paired Student's $t$ test or one-way analysis of variance followed by the Newman-Keuls multiple comparison test. For data not normally distributed, we applied the Kruskal-Wallis test followed by Dunn's multiple comparison test. Data were expressed as mean \pm SEM and significance was set at $p<$ 0.05 .

\section{Results}

DHEA treatment resulted in both reproductive and metabolic abnormalities in PCOS-like rats

To verify the potential relationship among hyperandrogenism, insulin resistance, and the circadian clock, DHEA was used as a trigger in female SD rats for 4 weeks. The DHEA group exhibited acyclicity, whereas the control group exhibited regular and complete estrous cycles in the last 8 days before decapitation (Fig. 1a). Moreover, the number of corpora lutea was decreased, whereas that of cystic follicles was increased in the ovaries of the DHEA group rats than in those of the control group rats (Fig. 1b). DHEA group rats showed a decreasing tendency in ovarian weight after 4-week treatment (Fig. 1d). However, no change was detected in the body weight between 2 groups during the whole process of rat model building (Fig. 1c). The higher area under the curve for GTT (Fig. 1e), increased serum leptin levels (Fig. 1f), and decreased serum adiponectin levels (Fig. 1g) after 4 weeks of DHEA treatment indicated impaired glucose metabolism and insulin sensitivity in DHEA group rats. Meanwhile, serum testosterone levels (Fig. 1h) as well as serum LH levels and LH/FSH ratio (Fig. 1i) were higher in this group. The combined findings of the examination of estrous cycle, ovarian histology, GTT, and serum testosterone and LH levels suggested that DHEA treatment induced a rat model 
Table 1 The primer sequences of the RT-qPCR tested genes

\begin{tabular}{|c|c|c|}
\hline Gene & Primer forward (5' to $\left.3^{\prime}\right)$ & Primer reverse ( $5^{\prime}$ to $3^{\prime}$ ) \\
\hline Bmal1 (rat) & GGCTGTTCAGCACATGAAAAC & GCTGCCCTGAGAATTAGGTGTT \\
\hline Clock (rat) & CTTCCTGGTAACGCGAGAAAG & GTCGAATCTCACTAGCATCTGAC \\
\hline Perl (rat) & GATGTGGGTGTCTTCTATGGC & AGGACCTCCTCTGATTCGGC \\
\hline Per2 (rat) & CAGGTTGAGGGCATTACCTCC & AGGCGTCCTTCTTACAGTGAA \\
\hline Cryl (rat) & CACTGGTTCCGAAAGGGACTC & CTGAAGCAAAAATCGCCACCT \\
\hline Cry2 (rat) & CACTGGTTCCGCAAAGGACTA & CCACGGGTCGAGGATGTAGA \\
\hline Sirt1 (rat) & TGATTGGCACCGATCCTCG & CCACAGCGTCATATCATCCAG \\
\hline Nampt (rat) & CCTGGTATCCAATTACAGTGGC & CCAAATGAGCAGATGCCCCTAT \\
\hline Glut4 (rat) & ACACTGGTCCTAGCTGTATTCT & CCAGCCACGTTGCATTGTA \\
\hline Pparg (rat) & GGAAGACCACTCGCATTCCTT & GTAATCAGCAACCATTGGGTCA \\
\hline$\beta$-Actin (rat) & GGCCAACCGTGAAAAGATGACC & AACCCTCATAGATGGGCACAG \\
\hline Bmal1 (mouse) & ACAGTCAGATTGAAAAGAGGCG & GCCATCCTTAGCACGGTGAG \\
\hline Clock (mouse) & ATGGTGTTTACCGTAAGCTGTAG & CTCGCGTTACCAGGAAGCAT \\
\hline Per1 (mouse) & GAATTGGAGCATATCACATCCGA & CCCGAAACACATCCCGTTTG \\
\hline Per2 (mouse) & CTCCAGCGGAAACGAGAACTG & TTGGCAGACTGCTCACTACTG \\
\hline Sirt1 (mouse) & ATGACGCTGTGGCAGATTGTT & CCGCAAGGCGAGCATAGAT \\
\hline Nampt (mouse) & GCAGAAGCCGAGTTCAACATC & TITCACGGCATTCAAAGTAGGA \\
\hline Glut4 (mouse) & GGACCGGATTCCATCCCAC & TCCCAACCATTGAGAAATGATGC \\
\hline Pparg (mouse) & CTCCAAGAATACCAAAGTGCGA & GCCTGATGCTITATCCCCACA \\
\hline$\beta$-Actin (mouse) & GTGACGTTGACATCCGTAAAGA & GCCGGACTCATCGTACTCC \\
\hline
\end{tabular}

with PCOS-like clinical symptoms, especially in a state of insulin resistance [16].

Disrupted rhythmic expression of circadian clock genes and insulin resistance-related genes was detected in the liver tissue of PCOS-like rats

We investigated circadian genes and insulin pathways in the liver tissue of PCOS-like rats. Sirtuin 1 (Sirt1) and nicotinamide phosphoribosyltransferase (Nampt) are the rhythmically expressed genes closely associated with insulin resistance $[17,18]$. Bmal1 mRNA expression decreased in the liver of PCOS-like rats compared with that of control rats, with the most significant reduction at ZT10. Moreover, Per2 mRNA expression displayed a tendency to decrease at ZT15 and Cry 2 mRNA expression significantly increased at ZT10 and ZT20, whereas no difference was found in the expression of other circadian clock genes (Fig. 2a). The decreased BMAL1 and P$\mathrm{AKT}$ protein expression in the liver implied the association between circadian clock genes and the insulinresistant state in PCOS (Fig. 2b), which was further supported by the disrupted rhythmic mRNA expression of Sirt1, Nampt, Glut4, and peroxisome proliferator activated receptor gamma (Pparg). Specifically, Sirt1 mRNA expression in PCOS-like rats decreased at ZT0 and increased at ZT15, respectively. Nampt mRNA expression increased and Glut4 mRNA expression decreased at
ZT5. Furthermore, Pparg mRNA expression decreased at ZT5 and increased at ZT10, respectively (Fig. 2c).

Disrupted rhythmic expression of circadian clock genes and insulin resistance-related genes was detected in the adipose tissue of PCOS-like rats

We also explored circadian gene expression in the adipose tissue of PCOS-like rats. Bmal1 mRNA expression in PCOS-like rats significantly decreased at ZT5, whereas Clock mRNA expression increased at ZT20 (Fig. 3a). Adipose tissue of PCOS-like rats also showed a reduction in BMAL1 and $\mathrm{P}-\mathrm{AKT}$ protein expression (Fig. 3b). A difference in the mRNA expression of Sirt1, Nampt, and Pparg, but not Glut4, in adipose tissue was discovered between PCOS-like rats and control rats. Specifically, Nampt mRNA expression in PCOS-like rats was decreased at ZT0 and increased at ZT10 and ZT15. Both Sirt1 and Pparg mRNA expressions were increased at ZT10 (Fig. 3c).

Hyperandrogenism facilitated BMAL1-mediated insulin resistance through the NAMPT/NAD ${ }^{+} / \mathrm{SIRT}_{1}$ pathway in mature adipocytes

In our previous work, we have certified that testosterone treatment decreases BMAL1 expression, and further reduces glucose uptake by inhibiting the NAMPT/NAD ${ }^{+}$/ SIRT1 pathway and GLUT4 expression in HepG2 cells 


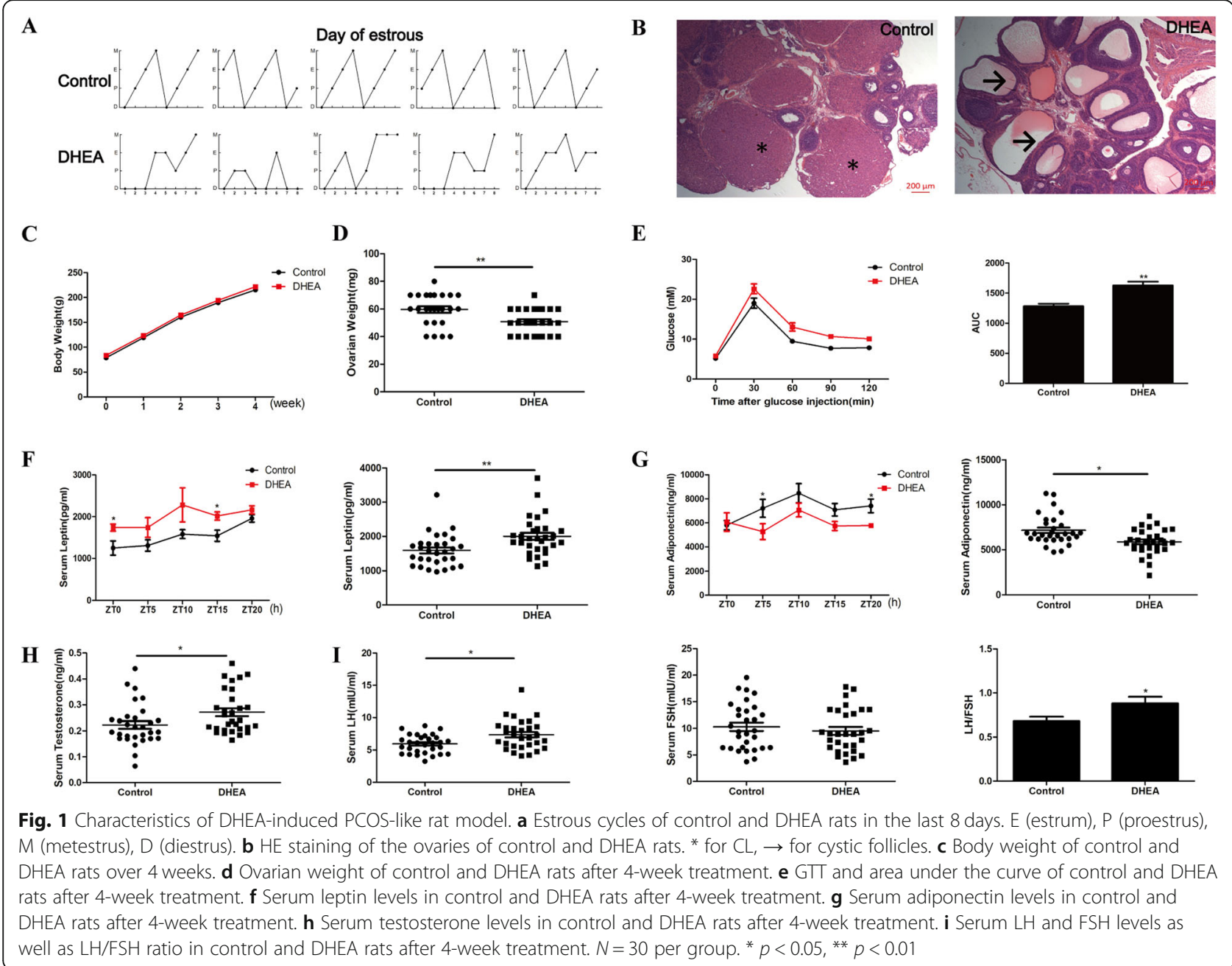

[10]. Mature adipocytes induced by the 3T3-L1 cell line are always used as the cell model for molecular research studies conferring to adipose tissue. Therefore we used this cell line to explore the mechanism of association between BMAL1 and insulin resistance in adipose tissues. Oil red $\mathrm{O}$ staining was used to identify the mature adipocytes (Fig. 4a).

We found that knocking down Bmal1 or Nampt decreased glucose uptake in mature adipocytes (Fig. 4b). NAMPT-mediated $\mathrm{NAD}^{+}$biosynthesis plays a critical role in numerous biological processes through the $\mathrm{NAD}^{+}$-dependent deacetylase SIRT1 [19]. Consequently, we further verified the regulation of BMAL1 and the NAMPT/NAD ${ }^{+} / \mathrm{SIRT} 1$ pathway. Knocking down Bmal1 decreased Nampt, Sirt1, and Glut4 mRNA expression as well as cellular NAD $^{+}$ levels in mature adipocytes (Fig. 4c, d). Moreover, knocking down Nampt or Sirt1 decreased Glut4 mRNA expression without any effect on Bmal1 expression (Fig. 4e, f). Therefore, Bmal1 knockdown was suggested to reduce glucose uptake through the inhibition of the NAMPT/NAD ${ }^{+} / \mathrm{SIRT} 1$ pathway and Glut4 expression in mature adipocytes.

Additionally, Bmal1, Nampt, and Sirt1 mRNA expressions were decreased in a dose-dependent manner in mature adipocytes after testosterone treatment, whereas Clock, Per1 and Per2 mRNA expressions unchanged (Fig. 4g). Treatment with testosterone also decreased glucose uptake in mature adipocytes (Fig. 4b). Testosterone treatment partly hindered the increased NAMPT/ $\mathrm{NAD}^{+} /$SIRT1 pathway and Glut4 expression after Bmal1 overexpression. Concurrently, compared with the control group, the addition of testosterone after Bmall overexpression did not decrease the expression of downstream factors as testosterone treatment alone did (Fig. 4h). Thus, hyperandrogenism might facilitate insulin resistance via BMAL1-mediated pathway in mature adipocytes.

\section{Discussion}

In this study, we demonstrated that DHEA-induced PCOS-like rats exhibited insulin resistance as well as 


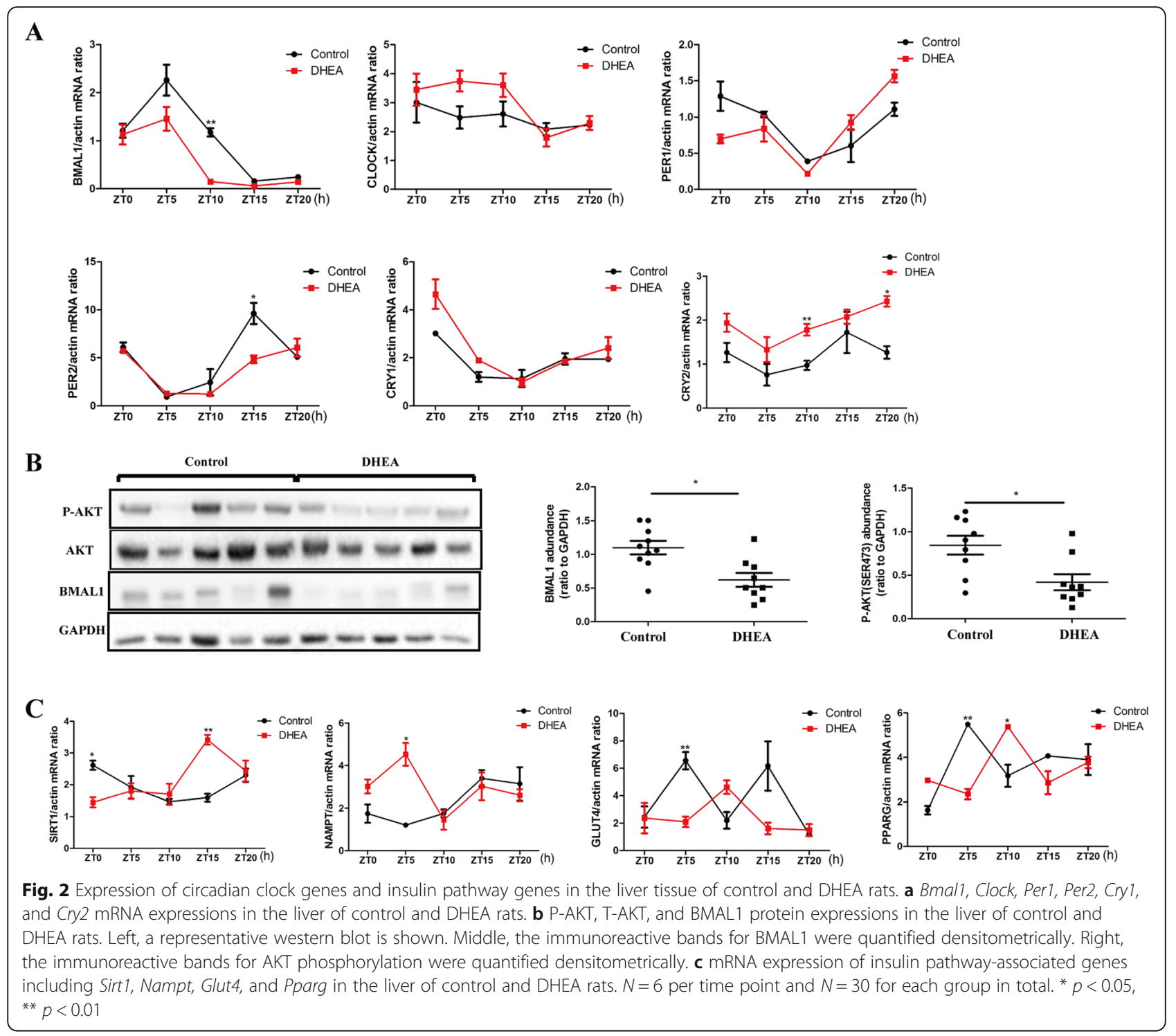

arrhythmic expression of circadian clock genes in the liver and adipose, particularly showing decreased BMAL1 expression. Meanwhile, hyperandrogenism led to the negative regulation of BMAL1-induced expression of NAMPT/NAD ${ }^{+} /$SIRT1 pathway, further inhibiting downstream GLUT4 and contributing to insulin resistance in mature adipose cells, which was consistent with our previous results in HepG2 cells [10]. Thus, our study uncovered the potentially novel role of BMAL1 in the contribution of hyperandrogenism to insulin resistance in PCOS.

Environmental risk factors combined with genetic factors give rise to hyperandrogenism and insulin resistance, both of which are known to be involved in the onset of PCOS [20]. Circadian disruption, especially constant darkness, induces insulin resistance of PCOS in rats, including increased fasting blood glucose level and increased serum insulin and leptin levels [10]. To investigate the relationship between circadian dysfunction and glucose metabolism, we used liver and adipose tissues of the DHEA-induced PCOS-like rat model as these are the two principal tissues involved in insulin resistance. Rhythmic disorder in the expression of circadian genes, especially Bmal1, as well as insulin pathway genes was observed in both liver and adipose of PCOS-like rats. Our previous study has shown the decreased BMAL1 mRNA expression in PCOS patients [10]. BMAL1 plays a vital role in lipid and glucose metabolism. It promotes de novo lipogenesis through insulinmammalian target of rapamycin complex 2 (mTORC2)AKT signaling in liver tissue [21], and Bmal1-null male and female mice have been reported to display increased adiposity [22]. BMAL1 knockdown induces insulin resistance, as indicated by markedly impaired insulin- 


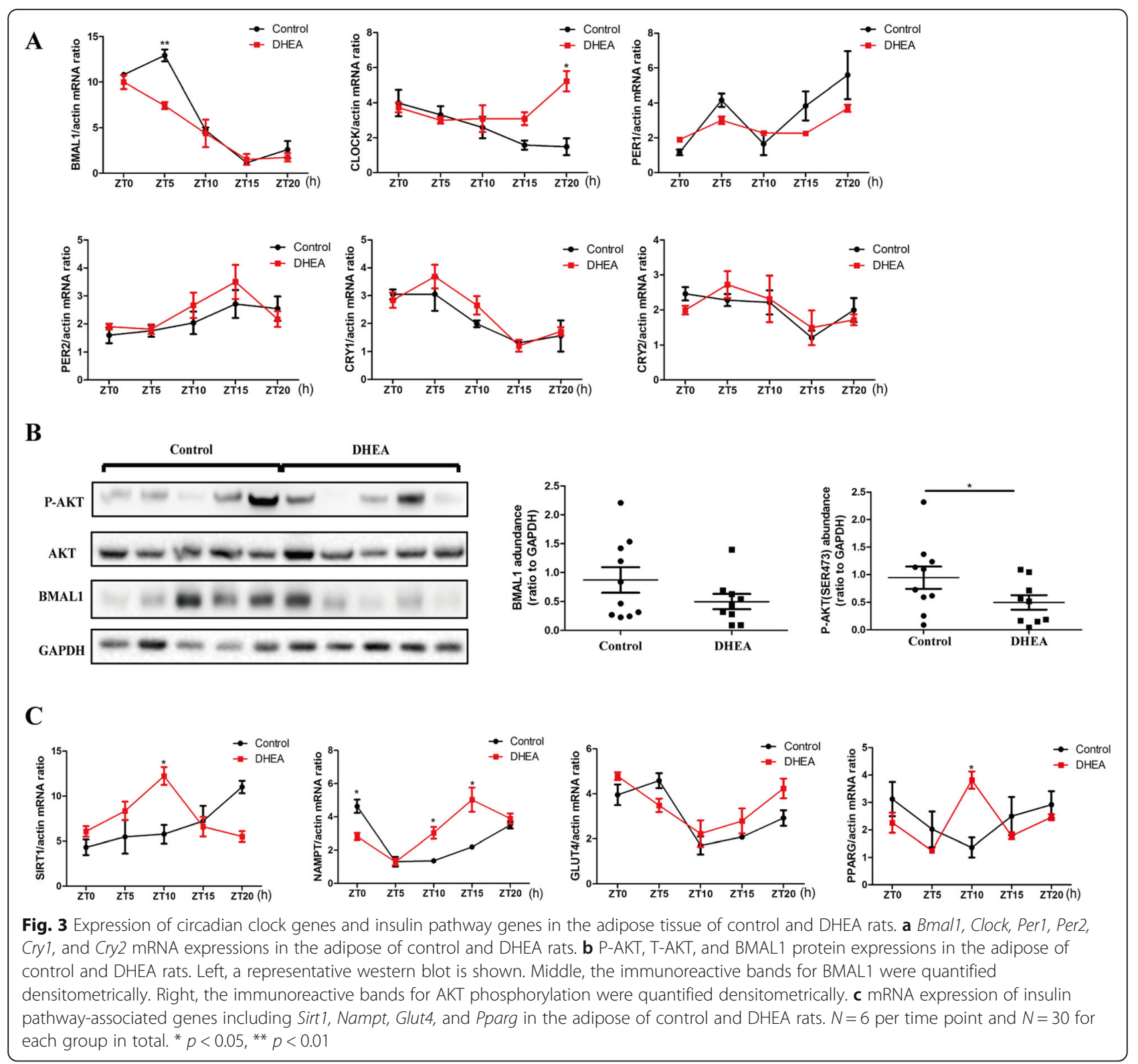

stimulated phosphorylation of insulin receptor and AKT pathway [18]. BMAL1 is also involved in estrogen synthesis via SIRT1 in PCOS patients [23]. Hence the change in BMAL1 expression plays a potential role in hyperandrogenism-induced insulin resistance in PCOS.

As a circadian upstream factor of SIRT1, NAMPT is involved in insulin resistance through PPAR $\gamma$ and adiponectin [17]. NAMPT and $\mathrm{NAD}^{+}$display circadian oscillations, and the circadian transcription factor CLOCK upregulates NAMPT [17]. Furthermore, adipose-specific NAMPT is closely related to insulin resistance in the adipose tissue, liver, and skeletal muscle through the regulation of PPARY and adiponectin [24]. Intelectin-1 promotes steroidogenesis in the granulosa cells of PCOS patients via NAMPT [25]. Previous study has confirmed the importance of the NAMPT/NAD ${ }^{+} / \mathrm{SIRT} 1$ pathway in the systemic regulation of glucose metabolism [26]. Based on the arrhythmic Nampt and Sirt1 mRNA expressions in the liver and adipose of PCOS-like rats, we speculated that NAMPT and SIRT1 might be the bridge linking BMAL1 and insulin resistance in PCOS patients. Using mature adipocytes, we confirmed that hyperandrogenism inhibited the BMAL1-induced NAMPT/ $\mathrm{NAD}^{+}$/SIRT1 pathway, and further reduced GLUT4 to suppress insulin sensitivity, which was consistent with our previous data in HepG2 cells (Figure S1) [10].

The effect of hyperandrogenism on circadian organization and clock function may be somewhat ubiquitous and represents a general impact of elevated androgen levels on circadian timing. Hyperandrogenism 


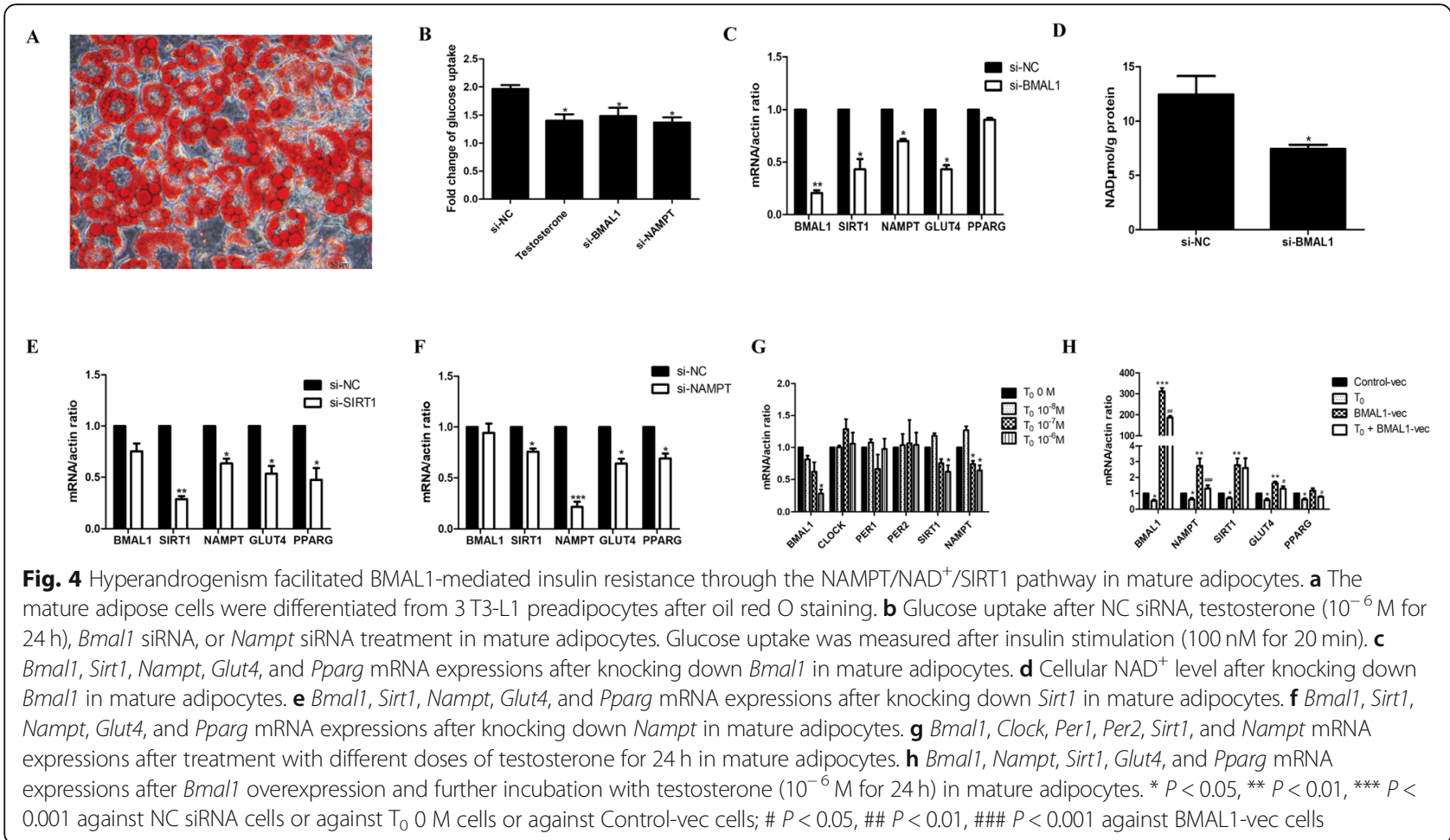

might affect the amplitude of clock output and the precision of clock control over behavior, but it might not affect circadian rhythms of clock gene expression in the suprachiasmatic nucleus [27]. Androgen receptor (AR) expression in suprachiasmatic nucleus neurons is not rhythmic, but it is significantly affected by circulating androgen levels. DHEA-induced growth suppression of preadipocytes is mediated via AR [28]. It is possible that AR expression in the liver and adipose tissues of PCOSlike rats might be differentially altered. Notably, AR expression in the liver and adipose tissues is closely associated with visceral fat mass and glucose homeostasis [29]. Thus, the association between AR signaling and BMAL1 in each tissue is also worth investigating in the future.

Among the limitations of this study is the fact that we did not detect the expression of circadian clock genes in the liver and adipose tissues of women with or without PCOS due to the scarcity of samples. Secondly, since we proposed and confirmed the role of BMAL1 in the contribution of hyperandrogenism to insulin resistance in PCOS, it might be a novel potential therapeutic target for PCOS patients. More experiments with feasible intervention such as melatonin treatment are urgently needed, and more experiments elaborating on the related molecular mechanisms will be required.

\section{Conclusions}

We investigated the changes in circadian clock genes expression and their association with hyperandrogenism and insulin resistance, putting forward a new molecular mechanism to elaborate the contribution hyperandrogenism to insulin resistance in PCOS. Hyperandrogenism resulted in the negative regulation of BMAL1induced expression of the NAMPT/NAD $/$ SIRT1 pathway, which further suppressed GLUT4, leading to insulin resistance in the liver and adipose tissues of rats. The disrupted rhythmic expression of circadian clock genes, specifically decreased BMAL1 expression, mediated the contribution of hyperandrogenism to insulin resistance in PCOS.

\section{Supplementary information}

Supplementary information accompanies this paper at https://doi.org/10. 1186/s12958-020-00592-1.

Additional file 1 Figure S1. Proposed signaling pathways

underpinning the essential role of BMAL1 in the contribution of hyperandrogenism to insulin resistance in PCOS.

\section{Abbreviations}

PCOS: Polycystic ovary syndrome; BMAL1: Brain and muscle ARNT-like protein 1; CLOCK: Circadian locomotor output cycles kaput; CRY: Cryptochrome; PER: Period; SD: Sprague-Dawley; DHEA: Dehydroepiandrosterone; GTT: Glucose tolerance test; HE: Hematoxylin and eosin; DMEM: Dulbecco's modified eagle medium; FBS: Fetal bovine serum; ELISA: Enzyme-linked immunosorbent assay; LH: Luteinizing hormone; FSH: Follicle-stimulating hormone; RT-qPCR: Real-time quantitative polymerase chain reaction; $\mathrm{NAD}^{+}$: Nicotinamide adenine dinucleotide; NAMPT: Nicotinamide phosphoribosyltransferase; PPARG: Peroxisome proliferator activated receptor gamma; SIRT1: Sirtuin 1; GLUT4: Glucose transporter type 4; AR: Androgen receptor 


\section{Acknowledgements}

We greatly acknowledge all research volunteers for participating in the study. We are deeply grateful to Jingwen Lang, Yang Wang for assistance with sample preparation of rats.

\section{Authors' contributions}

$J Z, S L, M H$, and YD contribute to the design of the experiment. JZ, SL, FD contribute to conducting the experiments. JZ, SL, MH, JL, and YD contribute to analyzing the data. JZ, SL, MH, FD, JL, YD finish drafting the article or revising it critically for important intellectual content. YD is responsible for the final approval of the version to be published. The author(s) read and approved the final manuscript.

\section{Funding}

This research was supported by grants from the National Key Research and Development Program of China (No. 2018YFC1003202 and 2017YFC1001002), National Natural Science Foundation (No. 81971343, 81671414, and 81901549), and Shanghai Commission of Science and Technology (No. 19410760300 and 17DZ2271100).

\section{Availability of data and materials}

The datasets generated during and/or analyzed during the current study are available from the corresponding author on reasonable request.

\section{Ethics approval}

The animal experiments were approved by the Committee on Laboratory Animal Research of Shanghai Jiaotong University, China.

\section{Consent for publication}

Not applicable.

\section{Competing interests}

The authors declare no competing or financial interests.

\section{Author details}

${ }^{1}$ Center for Reproductive Medicine, Ren Ji Hospital, School of Medicine, Shanghai Jiao Tong University, 845 Lingshan Road, Shanghai 200135, China. ${ }^{2}$ Shanghai Key Laboratory for Assisted Reproduction and Reproductive Genetics, Shanghai 200135, China. ${ }^{3}$ Community Health Service Center, Tianmu West Road, Jingan District, Shanghai 200041, China.

Received: 7 March 2020 Accepted: 15 April 2020

Published online: 25 April 2020

\section{References}

1. Elia E, Sander V, Luchetti CG, Solano ME, Di Girolamo G, Gonzalez C, et al. The mechanisms involved in the action of metformin in regulating ovarian function in hyperandrogenized mice. Mol Hum Reprod. 2006;12:475-81.

2. Stepto NK, Cassar S, Joham AE, Hutchison SK, Harrison CL, Goldstein RF, et al. Women with polycystic ovary syndrome have intrinsic insulin resistance on euglycaemic-hyperinsulaemic clamp. Hum Reprod. 2013;28: 777-84.

3. Dunaif A, Wu X, Lee A, Diamanti-Kandarakis E. Defects in insulin receptor signaling in vivo in the polycystic ovary syndrome (PCOS). Am J Physiol Endocrinol Metab. 2001;281:E392-9.

4. Merkin SS, Phy JL, Sites CK, Yang D. Environmental determinants of polycystic ovary syndrome. Fertil Steril. 2016;106:16-24.

5. Ueda HR, Hayashi S, Chen W, Sano M, Machida M, Shigeyoshi Y, et al. System-level identification of transcriptional circuits underlying mammalian circadian clocks. Nat Genet. 2005;37:187-92.

6. Pan X, Taylor MJ, Cohen E, Hanna N, Mota S. Circadian clock, time-restricted feeding and reproduction. Int J Mol Sci. 2020;21(3).

7. Mayeuf-Louchart A, Zecchin M, Staels B, Duez H. Circadian control of metabolism and pathological consequences of clock perturbations. Biochimie. 2017;143:42-50.

8. Vinogradova IA, Anisimov VN, Bukalev AV, Semenchenko AV, Zabezhinski MA. Circadian disruption induced by light-at-night accelerates aging and promotes tumorigenesis in rats. Aging (Albany NY). 2009;1:855-65.

9. Green CB, Takahashi JS, Bass J. The meter of metabolism. Cell. 2008;134: $728-42$.
10. Li S, Zhai J, Chu W, Geng X, Chen ZJ, Du Y. Altered circadian clock as a novel therapeutic target for constant darkness-induced insulin resistance and hyperandrogenism of polycystic ovary syndrome. Transl Res. 2020;219: $13-29$.

11. Spinedi E, Cardinali DP. The polycystic ovary syndrome and the metabolic syndrome: a possible chronobiotic-cytoprotective adjuvant therapy. Int J Endocrinol. 2018;2018:1349868.

12. Sellix MT, Murphy ZC, Menaker M. Excess androgen during puberty disrupts circadian organization in female rats. Endocrinology. 2013;154:1636-47.

13. Mereness AL, Murphy ZC, Sellix MT. Developmental programming by androgen affects the circadian timing system in female mice. Biol Reprod. 2015;92:88.

14. Simon SL, McWhirter L, Diniz BC, Bubar KM, Kaar JL, Pyle L, et al. Morning circadian misalignment is associated with insulin resistance in girls with obesity and polycystic ovarian syndrome. J Clin Endocrinol Metab. 2019;104: 3525-34.

15. Pan Y, Shu JL, Gu HF, Zhou DC, Liu XL, Qiao QY, et al. Erythropoietin improves insulin resistance via the regulation of its receptor-mediated signaling pathways in 3T3L1 adipocytes. Mol Cell Endocrinol. 2013;367:116-23.

16. Caldwell AS, Middleton $\amalg$, Jimenez M, Desai R, McMahon AC, Allan CM, et al. Characterization of reproductive, metabolic, and endocrine features of polycystic ovary syndrome in female hyperandrogenic mouse models. Endocrinology. 2014;155:3146-59.

17. Stromsdorfer KL, Yamaguchi S, Yoon MJ, Moseley AC, Franczyk MP, Kelly SC, et al. NAMPT-mediated NAD(+) biosynthesis in adipocytes regulates adipose tissue function and multi-organ insulin sensitivity in mice. Cell Rep. 2016;16:1851-60.

18. Zhou B, Zhang Y, Zhang F, Xia Y, Liu J, Huang R, et al. CLOCK/BMAL1 regulates circadian change of mouse hepatic insulin sensitivity by SIRT1. Hepatology. 2014;59:2196-206.

19. Revollo JR, Grimm AA, Imai S. The NAD biosynthesis pathway mediated by nicotinamide phosphoribosyltransferase regulates Sir2 activity in mammalian cells. J Biol Chem. 2004;279:50754-63.

20. Bednarska S, Siejka A. The pathogenesis and treatment of polycystic ovary syndrome: What's new? Adv Clin Exp Med. 2017;26:359-67.

21. Zhang D, Tong X, Arthurs B, Guha A, Rui L, Kamath A, et al. Liver clock protein BMAL1 promotes de novo lipogenesis through insulin-mTORC2-AKT signaling. J Biol Chem. 2014;289:25925-35.

22. Kennaway DJ, Varcoe TJ, Voultsios A, Boden MJ. Global loss of bmal1 expression alters adipose tissue hormones, gene expression and glucose metabolism. PLoS One. 2013;8:e65255.

23. Zhang J, Liu J, Zhu K, Hong Y, Sun Y, Zhao X, et al. Effects of BMAL1-SIRT1positive cycle on estrogen synthesis in human ovarian granulosa cells: an implicative role of BMAL1 in PCOS. Endocrine. 2016;53:574-84.

24. Ramsey KM, Yoshino J, Brace CS, Abrassart D, Kobayashi Y, Marcheva B, et al. Circadian clock feedback cycle through NAMPT-mediated NAD+ biosynthesis. Science. 2009;324:651-4.

25. Cloix L, Reverchon M, Cornuau M, Froment P, Rame C, Costa C, et al. Expression and regulation of INTELECTIN1 in human granulosa-lutein cells: role in IGF-1-induced steroidogenesis through NAMPT. Biol Reprod. 2014;91:50.

26. Imai S, Yoshino J. The importance of NAMPT/NAD/SIRT1 in the systemic regulation of metabolism and ageing. Diabetes Obes Metab. 2013;15(Suppl 3):26-33.

27. Karatsoreos IN, Wang A, Sasanian J, Silver R. A role for androgens in regulating circadian behavior and the suprachiasmatic nucleus. Endocrinology. 2007;148:5487-95.

28. Fujioka K, Kajita K, Wu Z, Hanamoto T, Ikeda T, Mori I, et al. Dehydroepiandrosterone reduces preadipocyte proliferation via androgen receptor. Am J Physiol Endocrinol Metab. 2012;302:E694-704.

29. De Gendt K, Verhoeven G. Tissue- and cell-specific functions of the androgen receptor revealed through conditional knockout models in mice. Mol Cell Endocrinol. 2012;352:13-25.

\section{Publisher's Note}

Springer Nature remains neutral with regard to jurisdictional claims in published maps and institutional affiliations. 